\author{
Ecología
}

\title{
Escarabajos copronecrófagos (Scarabaeidae: Scarabaeinae) de la Reserva Natural Educativa Colonia Benítez, Chaco, Argentina
}

\author{
Copronecrophagous scarab beetles (Scarabaeidae: Scarabaeinae) from Colonia Benitez Educative \\ Natural Reserve, Chaco, Argentina
}

\author{
Mario G. Ibarra-Polesel*, Miryam P. Damborsky y Eduardo Porcel \\ Facultad de Ciencias Exactas y Naturales y Agrimensura, Universidad Nacional del Nordeste, Av. Libertad 5470, 3400 Corrientes, Argentina
}

Recibido el 26 de agosto de 2014; aceptado el 26 de febrero de 2015

Disponible en Internet el 29 de julio de 2015

\begin{abstract}
Resumen
Se describe la composición de escarabeinos de la Reserva Natural Educativa Colonia Benítez. Se realizaron 6 muestreos en bosque de quebracho y selva en galería en periodo seco y lluvioso. Los escarabajos se recolectaron mediante trampas cebadas con materia fecal y calamar, y con trampas de luz. Se recolectaron 3,369 ejemplares de 26 especies. Eurysternus aeneus, Canthon quinquemaculatus, Canthon chalybaeus, Deltochilum komareki y Canthidium (Canthidium) sp., representaron el 65\% del total de los individuos. Los estimadores no paramétricos de riqueza indican un porcentaje alto de completitud del inventario. La estructura del ensamble varió significativamente entre periodos, diferentes tipos de cebo y en los meses de muestreo, no así entre ambientes. En épocas secas, la abundancia y diversidad fueron mayores que en el periodo lluvioso. En marzo del 2011 se registró la mayor riqueza, diversidad y abundancia. Mediante trampas cebadas con materia fecal se obtuvo la mayor riqueza y con las de calamar la mayor abundancia. La temperatura máxima fue la variable que mejor explicó los cambios en la composición del ensamble. La riqueza observada demuestra que incluso pequeñas zonas boscosas son importantes áreas de conservación. Este es el primer estudio de la diversidad de Scarabaeinae en el Sitio Ramsar Humedales Chaco.

Derechos Reservados @ 2015 Universidad Nacional Autónoma de México, Instituto de Biología. Este es un artículo de acceso abierto distribuido bajo los términos de la Licencia Creative Commons CC BY-NC-ND 4.0.
\end{abstract}

Palabras clave: Diversidad; Coleoptera; Área protegida; Ambientes nativos; Noreste argentino

\begin{abstract}
Scarabaeinae assemblages composition from Colonia Benitez Reserve are assessed. Six samples using pitfalls, baited with squid and dung, and light traps, were carried out in dry and rainy seasons in 2 environments. A total of 3,369 specimens were caught belonging to 26 species. Eurysternus aeneus, Canthon quinquemaculatus, Canthon chalybaeus, Deltochilum komareki and Canthidium sp. represented 65\% of all captures. Non-parametric richness estimators showed a reliable inventory. Significant differences in the Scarabaeinae assemblages structure was observed between periods, among baits and between sampled months, but not among environments. Abundance and diversity were slightly higher in the dry season. Richness, abundance and diversity were higher in the dryer period and in March (2011). More species were captured in excrement traps and more individuals in carrion traps. Maximum temperature was the variable that best explained the variation in the community structure. This is the first study of Scarabaeinae diversity in a protected area of the Ramsar Site Chaco Wetlands. The observed richness highlights the importance of maintaining even small areas for conservation purposes.

All Rights Reserved (C) 2015 Universidad Nacional Autónoma de México, Instituto de Biología. This is an open access item distributed under the Creative Commons CC License BY-NC-ND 4.0.
\end{abstract}

Keywords: Diversity; Coleoptera; Protected area; Native environments; Northeastern Argentine

\footnotetext{
* Autor para correspondencia.

Correo electrónico: mario.ibarrapolesel@gmail.com (M.G. Ibarra-Polesel).

La revisión por pares es responsabilidad de la Universidad Nacional Autónoma de México.
} 


\section{Introducción}

La modificación de los ecosistemas como consecuencia de las actividades humanas es un fenómeno global, pero la magnitud y características de los cambios difieren en distintas regiones. La pérdida y fragmentación de ambientes naturales por la expansión agrícola, la deforestación y el sobrepastoreo desencadenan problemas ambientales a diferentes escalas, alteran la estructura y funcionamiento de los ecosistemas y originan la pérdida de hábitats y una disminución en la biodiversidad (Cabido et al., 1999).

El aumento de la frontera agrícola en Sudamérica, como en otras partes del mundo, se produce principalmente por deforestación de bosques nativos. Argentina está enfrentando en las últimas décadas uno de los procesos de deforestación más fuertes de su historia que se manifiesta con mayor intensidad en la región Chaqueña (Adámoli, Ginzburg y Torrella, 2011). Una estrategia de conservación de estos y otros bosques tropicales ha sido la creación de reservas naturales que alberguen los ecosistemas naturales (Schnack y López, 2003). La Reserva Natural Educativa Colonia Benítez (RNECB) está integrada al sistema de áreas protegidas bajo la jurisdicción de la administración de parques nacionales de Argentina, y se ubica en la ecorregión del Chaco Húmedo. Esta región clasificada como vulnerable dado el incremento de la degradación de sus bosques, cuenta con tan solo el 3\% de su superficie protegida (Burkart, 2006). Dos aspectos relevantes caracterizan a la reserva; está rodeada de ambientes donde se desarrollan actividades agropecuarias y su superficie es reducida, sin embargo, resguarda relictos de bosque de quebracho y de selva en galería, ambientes típicos del Chaco Húmedo (Chebez, 2005). Su flora ha sido meticulosamente revelada, principalmente por Schulz (1957), y con respecto a la fauna de artrópodos, se estudió la diversidad de hormigas (GómezLutz y Godoy, 2010), arañas (Escobar, Ávalos y Damborsky, 2012) y termitas (Godoy, Laffont, Coronel y Etcheverry, 2013).

La estimación de la diversidad es una tarea compleja y requiere la búsqueda de estrategias, entre ellas el uso de bioindicadores. Los invertebrados son componentes significativos de los ecosistemas e indicadores adecuados de la biodiversidad, no solo por su abundancia, sino también por las múltiples funciones que desempeñan; sin embargo, durante un largo tiempo no fueron considerados en las estrategias de monitorización y conservación, tendencia que se ha modificado en los últimos años (Mc Geoch et al., 2011). El abordaje de aspectos relacionados a la biodiversidad se efectuó históricamente evaluando la fauna de algunos vertebrados y de fanerógamas presentes en un ecosistema (Oliver y Beattie, 1993). Kremen et al. (1993) mencionan que la diversidad y abundancia de artrópodos terrestres también pueden proveer una valiosa base de información para ser utilizada en las estrategias y manejos de reservas naturales. Los escarabajos copronecrófagos de la subfamilia Scarabaeinae han sido propuestos como un taxón indicador de biodiversidad y para monitorizar el impacto de alteraciones antrópicas en bosques tropicales, ya que son vulnerables a la modificación del hábitat, sus métodos de recolección están estandarizados y su historia natural y taxonomía son bien conocidas (Favila y Halffter, 1997; Halffter y Favila, 1993; Nichols et al., 2007; Spector, 2006).
Los Scarabaeinae son un grupo diverso, abundante y con distribución global, que resultan indispensables en el funcionamiento de los ecosistemas (Halffter y Matthews, 1966). Los escarabajos del estiércol están involucrados en una serie de procesos ecológicos, tales como el reciclaje de nutrientes y la aireación del suelo, ya que manipulan, relocalizan, consumen y reciclan heces de mamíferos y cadáveres en descomposición (Nichols et al., 2008). El conocimiento de la composición de un ensamble en una determinada localidad para lograr una estimación más aproximada de la diversidad de especies demanda la utilización de diferentes métodos de recolecta (Sackmann, 2006). De esta manera, de la técnica de muestreo empleada dependerá que ciertas especies sean más propicias a ser recolectadas que otras. El efecto temporal también influye en la probabilidad de captura; la actividad diferencial de las especies en las distintas estaciones del año determina que su presencia varíe según la fecha en que se efectúen los muestreos (Jiménez-Valverde y Hortal, 2003). Esta característica se evidencia también en los escarabeinos que muestran variaciones temporales en su actividad, incluso en periodos de tiempo cortos (Andresen, 2008).

En este trabajo se propone conocer la diversidad de escarabajos copronecrófagos y la variación temporal de las especies en 2 formaciones vegetales de la RNECB: la selva de galería y el bosque de quebracho. Nuestras hipótesis son: 1) la escasa superficie de la RNECB limita el establecimiento de comunidades importantes de vertebrados, por lo que la riqueza de escarabajos copronecrófagos es reducida en este sitio; y 2) la composición de especies y la estructura del ensamble de escarabajos copronecrófagos serán diferentes entre las formaciones vegetales que resguarda la RNECB y entre los meses muestreados.

\section{Materiales y métodos}

Los muestreos se efectuaron en la RNECB (27 $19^{\prime} 04^{\prime \prime} \mathrm{S}$, 58 57’00” O), departamento Primero de Mayo de la provincia del Chaco, Argentina (fig. 1). La RNECB se encuentra a $64 \mathrm{~m}$ de altitud, el clima es subtropical con un promedio de precipitación anual de 1,300 mm y una temperatura mínima promedio de $19^{\circ} \mathrm{C}$ y máxima de $35^{\circ} \mathrm{C}$ (Bruniard, 1999). Es parte de la terraza fluvial del valle del río Paraná, la pendiente es escasa y presenta algunos problemas de drenaje. Desde el punto de vista biogeográfico el área está situada en la región Neotropical, subregión Chaqueña, en el sector del Chaco Oriental o Chaco Húmedo (Ginzburg y Adámoli, 2006). A pesar de su tamaño tan reducido ( 7 ha), incluye una alta riqueza de alrededor de 280 especies de plantas (Ginzburg y Adámoli, 2006). Entre sus formaciones vegetales se distingue el bosque con dominio de Schinopsis balansae (quebracho) y la selva en galería, en la que se reconocen Holocalyx balansae (ibirá pepé), Enterolobium contortisiliqum (timbó), Ficus monckii (ibapoí), Phytolacca dioica (ombú), Peltophorum dubium (ibirá pitá) y Syagrus romanzoffiana (palmera pindó) (Chebez, 2005).

Aunque su escasa superficie impide el desarrollo de grandes poblaciones de animales, el grupo de aves está bien representado, seguido por los anfibios y reptiles (Chebez, 2005). Entre los mamíferos se pueden mencionar a Dasypus novemcinctus 


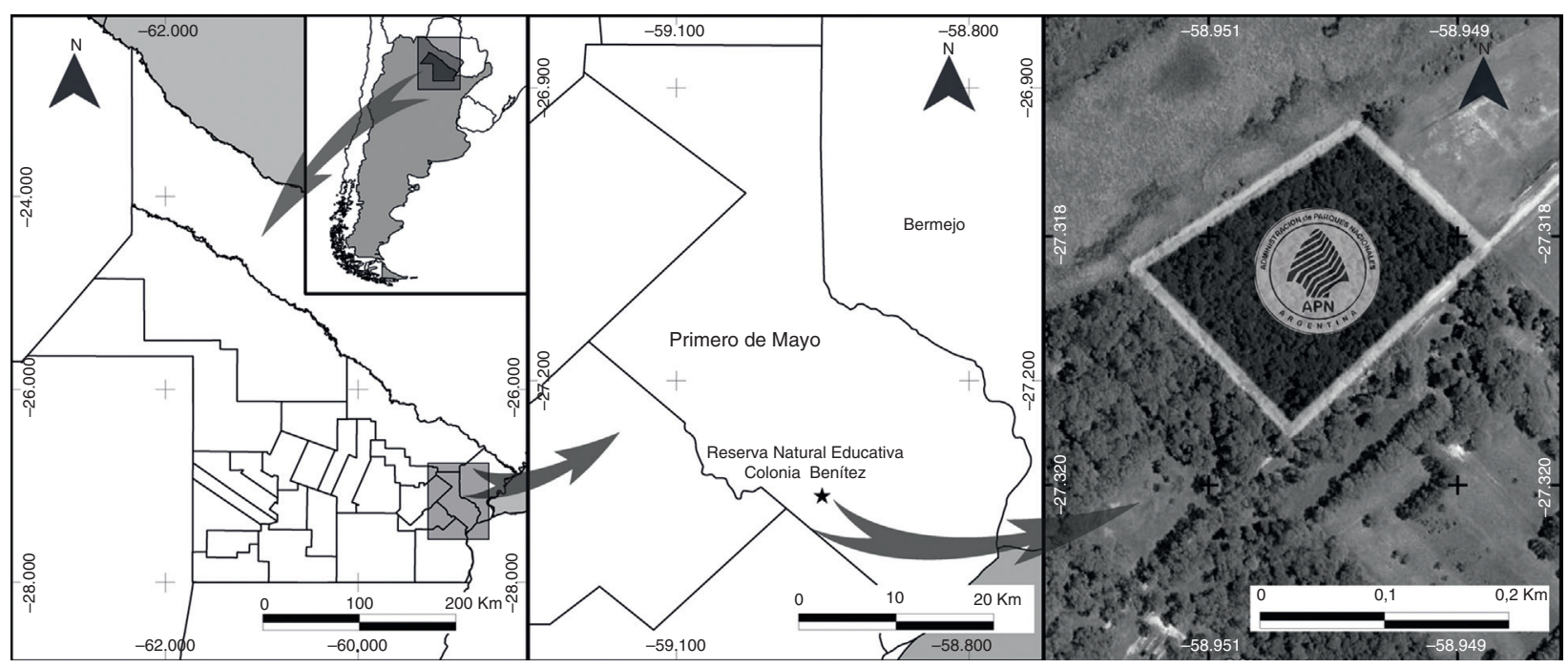

Figura 1. Localización de la RNECB, departamento Primero de Mayo, Chaco, Argentina.

(armadillo), Akodon cursor (ratón de hierba mostrador), Hydrochoerus hydrochaeris (carpincho o capybara), varias especies de murciélagos y el mono carayá Alouatta caraya (HeinonenFortabat y Chebez, 1997). En la RNECB no se practica la caza furtiva o la extracción de madera. Se encuentra dentro del predio que ocupa la Estación Experimental Agropecuaria Colonia Benítez del Instituto Nacional de Tecnología Agropecuaria (INTA), en donde se desarrollan estudios de producción pecuaria.

Se realizaron 6 muestreos, en estación lluviosa (diciembre de 2009, mayo y diciembre de 2010) y en estación seca (marzo y octubre de 2010 y marzo de 2011) en 2 formaciones vegetales, el bosque de quebracho y la selva en galería, empleando trampas de caída. En ambas unidades vegetales se ubicaron 2 transectos distanciados por $80 \mathrm{~m}$, en cada uno de ellos se instalaron 3 trampas separadas por $30 \mathrm{~m}$. Las trampas de un transecto se cebaron con materia fecal humana (coprotrampas) y las del otro con calamar en descomposición (necrotrampas). En total, se utilizaron 72 trampas en el estudio, las que permanecieron expuestas durante 3 días en cada muestreo, totalizando $432 \mathrm{~h}$ de trabajo de campo.

Cada trampa consistió de un recipiente descartable de $1,000 \mathrm{cc}(11 \mathrm{~cm}$ de diámetro superior $\times 12 \mathrm{~cm}$ de longitud), enterrado a ras del suelo en el que se colocó $20 \mathrm{~g}$ de cebo. Complementariamente se realizaron otros 5 muestreos con trampas de luz. Se instalaron 2 trampas tipo pantalla, separadas por una distancia de $50 \mathrm{~m}$, las cuales se mantuvieron activas durante $4 \mathrm{~h}$ a partir del crepúsculo. Se obtuvieron datos de temperaturas y precipitaciones de los registros de la estación meteorológica instalada en el INTA de Colonia Benítez. La temperatura promedio osciló entre $31.4^{\circ} \mathrm{C}$ en diciembre de 2009 y $11.5^{\circ} \mathrm{C}$ en mayo de 2010, con máximas y mínimas diarias que variaron entre los 37.6 y $4{ }^{\circ} \mathrm{C}$. La temperatura más baja a nivel del suelo se registró en mayo y la más elevada en diciembre de 2009. Se calificaron como lluviosos los meses en los cuales los registros pluviométricos superaron los $100 \mathrm{~mm}$, aquellos meses en los que el registro fue inferior se calificaron como secos (tabla 1). Los ejemplares recolectados se identificaron a nivel de especies, utilizando las claves de Edmonds y Zidek (2010), Génier (1996, 2009) y Vaz de Mello, Edmonds, Ocampo y Schoolmeesters (2011). Los ejemplares recolectados se depositaron en la colección del laboratorio de Biología de los Artrópodos de la Universidad Nacional del Nordeste (CARTROUNNE).

\section{Análisis de datos}

La eficiencia del muestreo en cada ambiente se evaluó mediante estimadores no paramétricos de riqueza: Chao1, Chao 2 y Jacknife de primer y segundo orden. Teniendo en cuenta la inexistencia de criterios objetivos para decidir si un inventario está lo suficientemente completo, se juzgó como un valor eficiente de muestreo cuando la riqueza observada fue equivalente al $80 \%$ de la riqueza estimada. Los estimadores no paramétricos se calcularon con el programa EstimateS versión 8.0 (Colwell, 2006).

También se estimó la cobertura del muestreo (Chao y Shen, 2003) para cada ambiente, ya que la representatividad de una muestra no solo depende de las especies que faltan, sino también de sus abundancias promedio (Moreno, Barragán, Pineda y Pavón 2011). Se utilizó el modelo multinomial (Shen, Chao y Lin, 2003) que permite predecir el número de nuevas especies que podrían ser capturadas en una futura recolecta de $m$ individuos. Para este modelo se utilizó el estimador de Shen et al. (2003), usando un punto de corte de 10 individuos. Estos 2 últimos análisis se realizaron con el programa SPADE (Chao y Shen, 2010). Se consideraron raras las especies con menos de 10 ejemplares; se identificaron las representadas por solo uno y por 2 individuos (Colwell, 2006).

La diversidad del ensamble de estercoleros recolectados con trampas cebadas se evaluó mediante el uso de número de especies efectivas propuesto por Jost (2006). Para este análisis se utilizó la diversidad de orden $0\left({ }^{0} D\right)$ o riqueza de especies; la diversidad de orden $1\left({ }^{1} D\right)$, que es el exponencial de la entropía 
Tabla 1

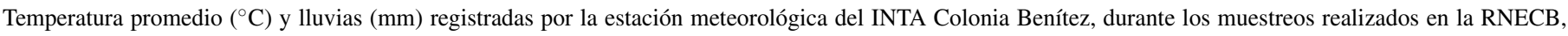
Chaco, Argentina, 2009-2011.

\begin{tabular}{|c|c|c|c|c|c|c|}
\hline \multirow[t]{2}{*}{ Meses } & \multicolumn{2}{|c|}{ Temperatura } & \multirow[t]{2}{*}{ Temperatura del suelo } & \multicolumn{2}{|c|}{ Lluvias registradas } & \multirow[t]{2}{*}{ Periodo } \\
\hline & Máxima & Mínima & & $\begin{array}{l}30 \text { días anteriores } \\
\text { al muestreo }\end{array}$ & $\begin{array}{l}14 \text { días anteriores } \\
\text { al muestreo }\end{array}$ & \\
\hline Dic-09 & 31.4 & 22.2 & 21.4 & 191.1 & 27.1 & Lluvioso \\
\hline Mar-10 & 31 & 21 & 18.5 & 97.9 & 16.9 & Seco \\
\hline May-10 & 20.9 & 11.5 & 9.5 & 178.7 & 158.8 & Lluvioso \\
\hline Oct-10 & 26 & 13.7 & 11.8 & 87.3 & 56.6 & Seco \\
\hline Dic-10 & 31.2 & 19.7 & 18.3 & 271.3 & 235.6 & Lluvioso \\
\hline Mar-11 & 30.1 & 19.4 & 17.2 & 67.5 & 0 & Seco \\
\hline
\end{tabular}

del índice de Shannon; y la diversidad de orden $2\left({ }^{2} D\right)$, que es el inverso del índice de Simpson. Los órdenes de estas medidas determinan qué tanto influyen las especies abundantes en la medida de la diversidad; ${ }^{\circ} D$ es completamente insensible a las abundancias de las especies, por lo tanto, equivale a la riqueza de especies; ${ }^{1} D$ todas las especies son incluidas con un peso proporcional a su abundancia en la comunidad $y,{ }^{2} D$ incluye solo las especies más abundantes (Gotelli y Chao, 2013; Moreno et al., 2011). La composición y la distribución de abundancia de las especies en cada unidad vegetal se contrastaron mediante curvas de rango-abundancia.

Para evaluar la disimilaridad de especies entre los 2 tipos de cebos utilizados se aplicó el índice de complementariedad (Colwell y Coddington, 1994). La preferencia alimentaria se analizó en especies con abundancia superior a 9 individuos a fin de minimizar el sesgo. Se calificaron como coprófagas o necrófagas cuando más del $80 \%$ de los individuos se recolectaron en copro- o necrotrampas, y generalistas, aquellas especies en las que menos del $80 \%$ de individuos se recolectaron en uno de los 2 tipos de trampa. Los Scarabaeinae fueron separados de acuerdo con su estrategia de relocalización del recurso en 3 grandes gremios: cavadores, rodadores y residentes (Halffter y Edmonds, 1982).

Para establecer si existen diferencias en la estructura del ensamble entre ambientes, periodos, meses de muestreo y tipos de cebos, se utilizó un análisis de varianza multifactorial PERMANOVA, con interacción basada en permutaciones multivariadas (Anderson, 2005), ya que se verificó que los datos no siguen una distribución normal. Se utilizó el procedimiento SIMPER para detectar las especies que caracterizaron a cada estación, se tuvo en cuenta aquellas especies que aportaron más del $10 \%$ de la abundancia. Con el propósito de evaluar el efecto de las variables ambientales sobre la composición del ensamble de escarabajos se aplicó el análisis Bioenv (Clarke y Ainsworth, 1993), el cual utiliza el coeficiente de correlación de rangos $r s$ de Spearman, donde valores cercanos a 1 indican que la variable estudiada es relevante. Las variables analizadas fueron: temperatura máxima, temperatura mínima, temperatura del suelo y lluvias registradas entre 14 y 30 días anteriores al retiro de las trampas. Ambos procedimientos se efectuaron con el programa PRIMER 5.0 (Clarke y Gorley, 2001). En todas las pruebas se consideró un nivel de significación $\alpha=0.05$.

\section{Resultados}

En las trampas cebadas se recolectaron 3,238 ejemplares y 21 especies. Eurysternus aeneus Génier fue la especie dominante, con una abundancia del $18.4 \%$ en relación con el total de los ejemplares capturados, seguido por Canthon quinquemaculatus Castelnau (13.1\%), Canthon chalybaeus Blanchard (11.7\%), Deltochilum komareki Balthasar(11.4\%) y Canthidium (Canthidium) sp. (10.2\%), que agruparon el $65 \%$ del total de individuos recolectados; estas especies, junto a Canthon chiriguano Martínez y Halffter, Onthophagus hirculus Mannerheim, Ontherus sulcator (Fabricius) y Anisocanthon villosus Harold, se capturaron en todos los muestreos.

Mediante trampas de luz se capturaron 131 individuos pertenecientes a 13 especies, de las cuales 5: Deltochilum valgum Burmeister, Ontherus (Ontherus) sp., Pseudocanthon xanthurus (Blanchard), Trichillum externepunctatum Borre y Trichillum heydeni (Harold), fueron capturadas exclusivamente con esta técnica. Asimismo, la especie más abundante y presente en todos los muestreos fue $O$. sulcator que representó el $71 \%$ del total de individuos. De esta forma, se capturaron con trampas cebadas y de luz 3,369 individuos pertenecientes a 26 especies agrupadas en 6 tribus y 14 géneros (tabla 2). Deltochilini fue la tribu mejor representada $(S=11)$, seguida por Coprini $(S=5)$. El género Canthon Hoffmannsegg reunió el mayor número de especies $(S=5)$. Se encontraron 10 especies calificadas por su abundancia como raras, de las cuales 6 estuvieron representadas por un solo individuo y 4 con abundancias entre 2 y 9 ejemplares (tabla 2).

\section{Representatividad del inventario}

De acuerdo con los estimadores no paramétricos Chao1 y Chao2, se alcanzó una eficiencia de captura del $95 \%$ en la selva en galería y del $100 \%$ en bosque de quebracho (fig. 2). En la selva, Jacknife de primer y segundo órdenes predijeron $23(87 \%)$ y $25(81 \%)$ especies respectivamente, y para el bosque de quebracho ambos estimaron 19 especies (95\%). La eficiencia de muestreo fue superior en el bosque de quebracho debido a la presencia de un mayor número de especies con un solo individuo en la selva. La cobertura de muestreo estimada para la selva y el bosque de quebracho fue del $C=0.998$ y $C=0.999$, 
Tabla 2

Escarabajos copronecrófagos recolectados mediante trampas cebadas con calamar y materia fecal y trampas de luz en bosque de quebracho y selva en galería de la RNECB, Chaco, Argentina, 2009-2011.

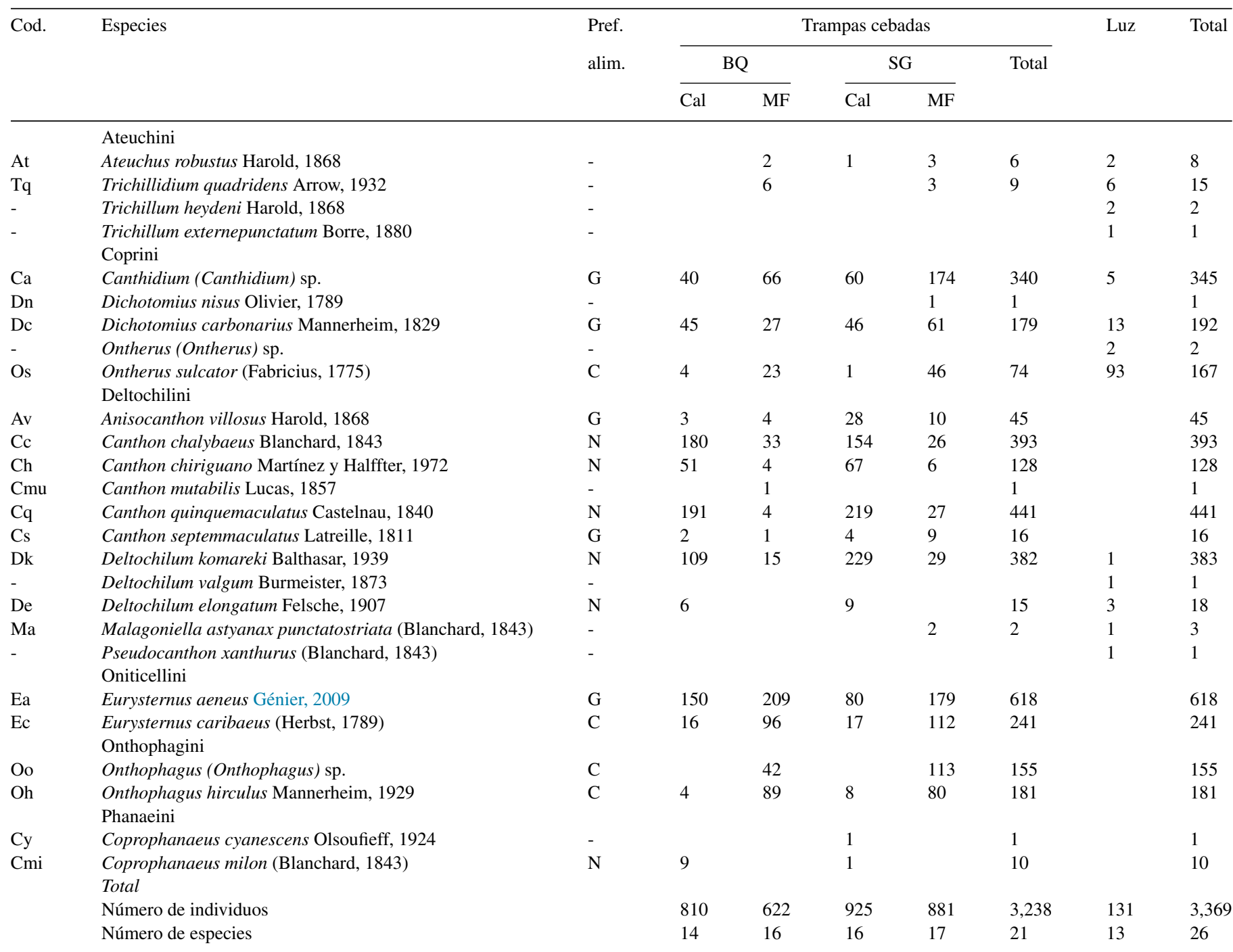

BQ: bosque de quebracho; Cal: calamar; C: coprófago; G: generalista; Luz: trampas de luz; MF: materia fecal; N: Necrófago; Pref. alim.: preferencia alimentaria; RNECB: Reserva Natural Educativa Colonia Benítez; SG: selva en galería.

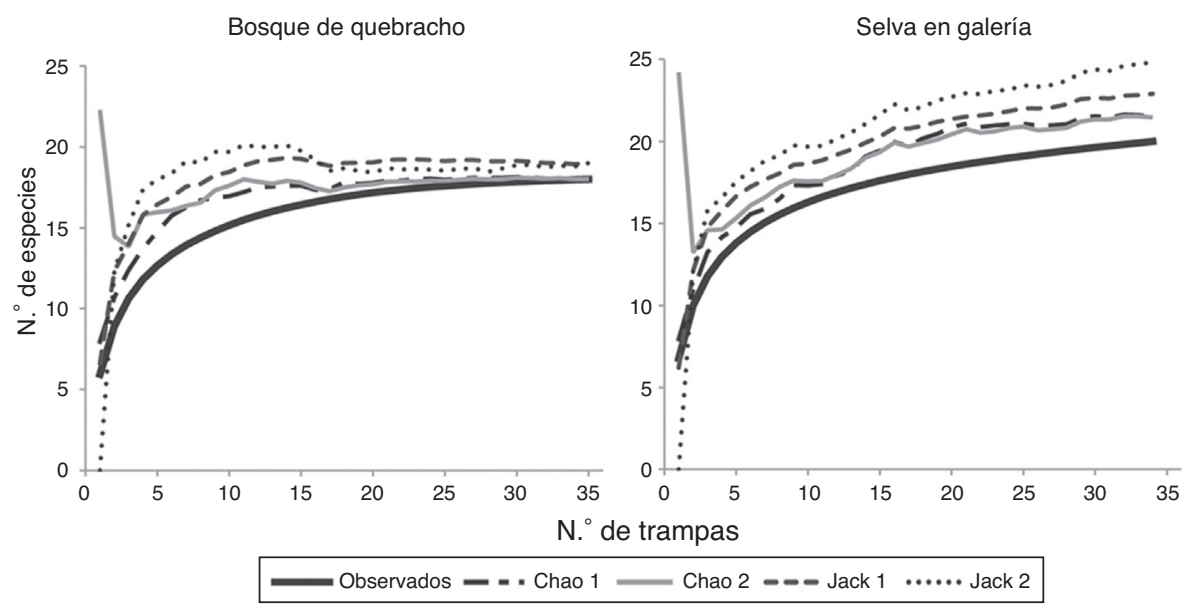

Figura 2. Curvas de acumulación de especies de Scarabaeinae recolectados mediante trampas cebadas en 2 unidades ambientales de la RNECB, Chaco, Argentina. 
Tabla 3

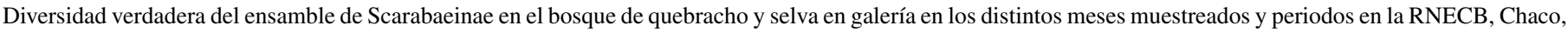
Argentina, 2009-2011.

\begin{tabular}{|c|c|c|c|c|c|}
\hline & & $\mathrm{n}$ & ${ }^{0} D$ & ${ }^{1} D$ & ${ }^{2} D$ \\
\hline \multirow[t]{2}{*}{ Ambientes } & Bosque de quebracho & 1,432 & 18 & 9.66 & 7.56 \\
\hline & Selva en galería & 1,806 & 20 & 11.15 & 9.62 \\
\hline \multirow[t]{6}{*}{ Meses } & Diciembre 2009 & 338 & 17 & 6.29 & 4.43 \\
\hline & Marzo 2010 & 548 & 15 & 5.42 & 3.82 \\
\hline & Mayo 2010 & 140 & 11 & 5.25 & 4.02 \\
\hline & Octubre 2010 & 367 & 14 & 8.44 & 6.77 \\
\hline & Diciembre 2010 & 586 & 16 & 6.36 & 4.85 \\
\hline & Marzo 2011 & 1,258 & 18 & 10.80 & 9.65 \\
\hline \multirow[t]{2}{*}{ Periodos } & Húmedo & 1,064 & 18 & 9.11 & 7.70 \\
\hline & Seco & 2,174 & 19 & 10.34 & 8.64 \\
\hline
\end{tabular}

${ }^{0} D$ : riqueza de especies; ${ }^{l} D$ : exponencial del índice de Shannon; ${ }^{2} D$ : inverso del índice de Simpson; RNECB: Reserva Natural Educativa Colonia Benítez.

respectivamente. El estimador de Shen et al. (2003) predijo que se necesitaría capturar otros 750 individuos para recolectar una nueva especie en la selva; mientras que para el bosque de quebracho, un incremento de captura de 1,500 ejemplares no asegura el hallazgo de otra especie.

\section{Estructura del ensamble}

Al comparar los ambientes estudiados, se registró en la selva una mayor abundancia, riqueza $\left({ }^{0} D\right)$ y diversidad $\left({ }^{1} D\right.$ y ${ }^{2} D$ ) que en el bosque de quebracho (tabla 3), y presentaron entre 8 y 9 especies muy abundantes. En ambos ambientes, los valores de diversidad ${ }^{l} D$ y ${ }^{2} D$ fueron relativamente similares. El análisis PERMANOVA no mostró diferencias significativas en la composición de Scarabaeinae entre ambientes $(F=1.92$, $\mathrm{p}=0.335$ ). Las curvas de rango-abundancia mostraron que en ambos ambientes las 6 especies más abundantes fueron las mismas, aunque el orden jerárquico fue distinto. Ambas curvas en su zona media y posterior mostraron una distribución similar, con pendientes poco pronunciadas. Sin embargo, la distribución fue más equitativa en la selva, donde 4 especies (Eurysternus aeneus [E. aeneus], Deltochilum komareki [D. komareki], Canthon quinquemaculatus [C. quinquemaculatus] y Canthidium (C.) sp.) fueron dominantes (fig. 3). En cambio, en el bosque se registró una pendiente pronunciada debido a una marcada abundancia de E. aeneus (25\%).

Al analizar los muestreos agrupados de acuerdo con el periodo en que se realizaron, se observó que durante el periodo seco el número de especies y diversidad fue levemente mayor que en el lluvioso, mientras que la abundancia fue 2 veces superior (tabla 3). Las diferencias entre periodos resultaron significativas $(F=7.41, \mathrm{p} 0.001)$. En cuanto a los meses estudiados, en marzo de 2011 se presentaron los máximos valores de abundancia, riqueza $\left({ }^{0} D\right)$ y diversidad $\left({ }^{1} D\right.$ y $\left.{ }^{2} D\right)$ (tabla 3 ). En contraposición, en las recolectas de mayo de 2010 se registraron los valores más bajos de los índices antes mencionados, con una pérdida del $51 \%$ de diversidad con respecto al mes de marzo de 2011. La diversidad ${ }^{2} D$ fue menor en el muestreo de marzo de 2010, debido a la dominancia de E. aeneus y Canthon chalybaeus (C. chalybaeus) que representaron en conjunto el $72 \%$ del total de individuos capturados en ese mes. Se

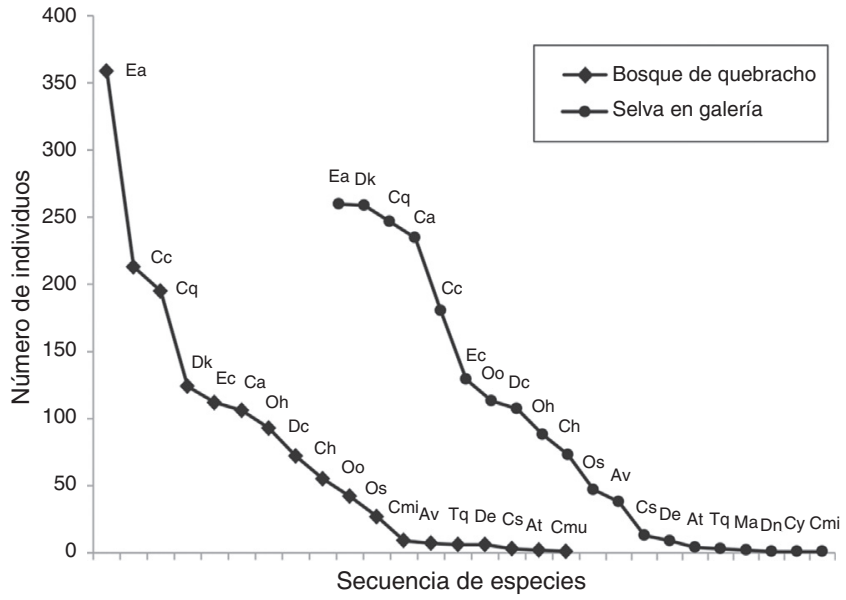

Figura 3. Curvas de rango-abundancia para las especies de Scarabaeinae recolectados mediante trampas cebadas en 2 unidades ambientales de la RNECB, Chaco, Argentina. (Cada punto en la curva representa una especie identificada con abreviatura (cod.), detallada en el tabla 2).

encontraron diferencias significativas entre meses de muestreo $(F=9.82, \mathrm{p}=0.001)$.

De acuerdo con el procedimiento SIMPER, las especies que contribuyeron con la similaridad de cada estación fueron: E. aeneus, Canthidium (C.) sp., E. caribaeus y D. carbonarius que caracterizaron al periodo lluvioso, y aportaron el $80 \%$ de la abundancia (tabla 4), esta última especie mostró marcados picos poblacionales en los meses húmedos, registrándose en los

\section{Tabla 4}

Porcentaje de similaridad (SIMPER) de especies recolectadas mediante trampas cebadas que caracterizan a cada una de las estaciones en la RNECB, Chaco, Argentina, 2010-2011.

\begin{tabular}{lcc}
\hline Especies & Seco & Húmedo \\
\hline $\begin{array}{l}\text { Eurysternus aeneus } \text { Génier, 2009 } \\
\text { Canthidium (C.) sp. }\end{array}$ & 10.00 & 9.61 \\
$\begin{array}{l}\text { Canthon chalybaeus } \text { Blanchard, 1843 } \\
\text { Eurysternus caribaeus (Herbst, 1789) }\end{array}$ & 7.43 & 9.42 \\
$\begin{array}{l}\text { Deltochilum komareki Balthasar, 1939 } \\
\text { Dichotomius carbonarius Mannerheim, 1829 }\end{array}$ & 4.89 & 5.30 \\
$\begin{array}{l}\text { Canthon quinquemaculatus } \text { Castelnau, 1840 } \\
\text { Similitud promedio }\end{array}$ & 3.07 & 3.44 \\
\hline
\end{tabular}


meses de diciembre (2009 y 2010) el $81 \%$ de su abundancia. E. aeneus, C. chalybaeus, D. komareki y C. quinquemaculatus caracterizaron al periodo seco y representaron el $75 \%$ de la abundancia.

\section{Preferencia alimentaria y gremios funcionales}

El análisis de preferencia alimentaria mostró que 6 especies fueron necrófagas, 5 generalistas y 4 coprófagas (tabla 2). Tres especies se comportaron como estenófagas, consumiendo exclusivamente excremento (Onthophagus sp.) o calamar (Coprophanaeus milon y Deltochilum elongatum). Los necrófagos reunieron el $40 \%$ de la abundancia al igual que los generalistas, el restante $20 \%$ correspondió a los de hábitos coprófagos. De las especies capturadas mediante trampas cebadas, 10 fueron cavadoras, 9 rodadoras y solo 2 endocópridas. El gremio de los rodadores fue el dominante, representando el $44 \%$ de los individuos recolectados, seguido por los cavadores (29.5\%) y los residentes (26.5\%). El 88\% del total de rodadores fue capturado con necrotrampas, mientras que con las mismas solo fueron atrapados el $31 \%$ de los residentes y el $23 \%$ de los cavadores. Las diferencias entre cebos resultaron significativas $(F=3.09, \mathrm{p}=0.007)$. El índice de complementariedad determinó un recambio del $38 \%$ entre ambos tipos de cebos.

\section{Variables ambientales}

De las variables analizadas en este estudio, las que mejor explicaron los cambios en la composición del ensamble fueron la temperatura máxima $(r=0.44)$, temperatura del suelo $(r=0.36)$ y la temperatura mínima promedio $(r=0.33)$. No se registraron correlaciones con las precipitaciones.

\section{Discusión}

El número de especies encontradas en este estudio en las trampas cebadas $(S=21)$, es semejante al encontrado por Damborsky, Bar, Álvarez-Bohle y Oscherov (2008) en otra área protegida de la provincia del Chaco, el Parque Provincial Pampa del Indio $(S=20)$, aunque en este último el esfuerzo de muestreo fue menor. La RNECB preserva una riqueza de escarabajos copronecrófagos que se mantiene en valores semejantes a los estimados para el Neotrópico (Halffter, Favila y Halffter, 1992).

\section{Representatividad del inventario}

A medida que el inventario se hace más completo, las probabilidades de encontrar una nueva especie disminuyen, mientras que el esfuerzo necesario para un nuevo hallazgo se eleva, pudiendo llegar a ser desproporcionadamente alto (Jiménez-Valverde y Hortal, 2003). En nuestro estudio, tanto los estimadores no paramétricos como los de cobertura de muestreo estimaron una riqueza muy cercana a la observada, lo que indica un inventario considerablemente fiable en ambos ambientes. De acuerdo con el estimador de Shen et al. (2003), en la selva es necesario recolectar en un futuro muestreo un número equivalente al $42 \%$ de los individuos ya recolectados para incrementar en tan solo un 5\% el inventario de escarabajos. En el bosque de quebracho, con un esfuerzo de muestreo aún superior, no se asegura el hallazgo de otra especie. De esta forma, se consideró innecesario seguir realizando muestreos, ya que la contribución al inventario sería muy baja, mientras que el esfuerzo y el costo para las poblaciones de escarabajos serían muy elevados, dado principalmente el pequeño tamaño de esta reserva.

Arellano (2002), en un estudio sobre metodologías de muestreo realizado en paisajes antropizados de Veracruz (México), menciona que utilizando otros tipos de cebos y otros métodos de recolecta (captura directa y trampas de intercepción de vuelo) se incrementaría en un 5\% el inventario de escarabeinos, dada la posibilidad de la existencia de unas pocas especies con hábitos tróficos especiales. Sin embargo, en relación con esta predicción, se resalta que en este trabajo las especies capturadas exclusivamente con trampas de luz $(S=5)$ contribuyeron con el $19 \%$ de la riqueza total de la RNECB $(S=26)$. Por lo tanto, se considera conveniente no incrementar el número de muestreos, pero sí utilizar una mayor variedad de técnicas.

Ontherus sulcator fue la única especie recolectada en todos los meses muestreados, con trampas de caída y trampas de luz; la atracción de esta especie hacia la luz artificial también es citada por Génier (1996). Cabrera-Walsh y Gandolfo (1996) identifican esta especie como representativa de los ambientes ganaderos del centro de Argentina, aunque también se cita en bosques de la región Neotropical (Campos y Hernández, 2013; Damborsky, Álvarez-Bohle, Ibarra-Polesel, Porcel y Fontana, 2015). Favila (2005) afirma que los fragmentos de bosque menores a 10 ha son invadidos por especies de pastizales circundantes; no es extraño entonces la presencia de $O$. sulcator en ambientes boscosos de la RNECB.

\section{Estructura del ensamble}

El análisis multivariado y las curvas de rango-abundancia no mostraron diferencias en la composición de escarabajos capturados en los distintos ambientes. Esta semejanza de los ensambles podría deberse a que entre la selva en galería y el bosque de quebracho no hay límites de separación definidos, y se desarrollan bajo un mismo dosel vegetal que permite un flujo constante de individuos. Asimismo, tanto en el bosque de quebracho como en la selva en galería, los valores entre la diversidad ${ }^{1} D$ y ${ }^{2} D$ no variaron considerablemente, lo que indica una composición equitativa de especies con abundancias altas e intermedias. En cambio, fue mayor la diferencia de los valores ${ }^{0} D$ con respecto a ${ }^{1} D$ y ${ }^{2} D$, debido al gran número de especies raras, las cuales se ven representadas hacia el final de las curvas de rangoabundancia. Este modelo, en el que se registran muchas especies raras, es característico de los bosques tropicales con estaciones secas y húmedas definidas (Halffter, 1991).

Los mayores valores de riqueza, abundancia y diversidad se obtuvieron en marzo de 2011, un mes de elevadas temperaturas y escasas lluvias. Se comprobó que la temperatura fue la variable que más influyó en el ensamble. Hernández y Vaz de Mello (2009), quienes trabajaron en áreas montañosas con predominio de bosque mesófilo, también demuestran la importancia de esta variable en el incremento del número de especies. 
No se registró correlación entre la composición del ensamble y las precipitaciones. Resultados similares han sido encontrados por Orozco y Pérez (2008) en 2 bosques (seco premontano y montañoso húmedo), aunque difieren con los de otros autores (Barbosa-Silva, Hernández, Ide y Moura, 2007; Bustos-Gómez y Lopera-Toro, 2003; Damborsky et al., 2015; Martínez, 2010; Milhomem, Vaz de Melo y Diniz, 2003) quienes encontraron la mayor riqueza y abundancia de especies durante los periodos lluviosos. Escobar (2000) destaca que las variaciones de la comunidad de escarabajos coprófagos entre periodos dependen también de los ambientes, y no encuentra relación entre la riqueza y los niveles de precipitación en bosques de montaña.

E. aeneus fue la única especie que caracterizó ambos periodos según el análisis SIMPER, debido a su abundancia en la mayoría de los meses muestreados. El conocimiento de los hábitos alimenticios de esta especie, recientemente descrita por Génier (2009), es escaso. Silva, Vaz de Mello y di Mare (2012) la citan como coprófaga, mientras que en este estudio, en el que se halló un mayor número de ejemplares, E. aeneus mostró hábitos generalistas con tendencia a la coprofagia. De las 4 especies que caracterizaron el periodo seco, 3 fueron rodadores $(C$. chalybaeus, C. quinquemaculatus y D. komareki). Esto podría deberse a que el gremio de los cavadores disminuye su actividad durante este periodo, ya que la compactación del suelo les dificulta la excavación de túneles (Janzen, 1983; Martínez, 2010).

\section{Preferencia alimentaria y gremios funcionales}

Con el uso de coprotrampas se colectó un mayor número de especies, mientras que con las necrotrampas el número de individuos fue superior. Estos resultados son similares a los obtenidos por Silva, Rosa-García y Vidal (2009a), mientras que BustosGómez y Lopera-Toro (2003), Milhomem et al. (2003), Silva y di Mare (2012) y Silva, Rosa-García y Vidal (2009b) encontraron un mayor número de especies e individuos con coprotrampas. En la RNECB las especies generalistas y necrófagas duplicaron en abundancia al grupo de coprófagos; esto se podría deber a que la oferta de excremento dentro de la RNECB es baja, el ganado no puede ingresar y el número de mamíferos es reducido, por lo que los animales muertos podrían estar constituyendo el recurso más frecuente, particularidad que es sobresaliente en la región Neotropical (Campos y Hernández, 2013; Halffter, 1991). Asimismo, las especies generalistas presentan una mejor capacidad de respuesta a la escasez de recursos, pudiendo utilizar otros alimentos, como hongos o frutos en descomposición (Hanski y Cambefort, 1991). Se identificaron un número similar de especies generalistas, necrófagas y coprófagas, lo que sugiere que la utilización equilibrada de los alimentos disponibles atenúa la competencia por el recurso y facilita la convivencia de las especies (Gill, 1991; Halffter y Halffter, 2009).

La mayor proporción de especies e individuos con hábitos cavadores y rodadores con respecto a los residentes es documentada por Barraza, Montes, Martínez y Deloya (2010) y Martínez, García, Pulido, Ospino y Narváez (2009). Esta característica se atribuye a la necesidad de los residentes de contar con gran cantidad de excremento (Halffter y Edmonds, 1982), recurso poco abundante en esta área. Asimismo, los suelos de la RNECB son de textura franco-limo-arcillosa (Ledesma, 1995), y es reconocida la afinidad de los cavadores por estas características edáficas (Hanski y Cambefort, 1991; MartínezHernández, Muñoz, Sierra-Quintero y Barraza-Méndez, 2012). Si bien el análisis de complementariedad indicó un bajo recambio de especies entre ambos tipos de cebos, el uso de diferentes atrayentes permitió la captura de especies estrictamente coprófagas y necrófagas. Así también, el uso de diferentes técnicas de captura, y la planificación de muestreos estacionales, contribuyeron para el logro de un inventario satisfactorio.

Pese a que la RNECB es un área reducida, el número de especies halladas en este trabajo refleja su buen estado de conservación. La RNECB no solo resguarda una muestra representativa de la fauna de escarabajos regional, sino también de los vertebrados de los cuales dependen. Por otro lado, este es el primer estudio de la diversidad de Scarabaeinae realizado en un área protegida del Sitio Ramsar Humedales Chaco. La caracterización del ensamble de escarabajos copronecrófagos en la RNECB resultaba necesaria, y constituye un punto de partida para la realización de estudios que permitirán una monitorización y un diagnóstico del impacto de las actividades humanas sobre la riqueza y diversidad de estercoleros en otras áreas. Desafortunadamente, la superficie protegida en el Chaco Húmedo se encuentra lejos de los estándares internacionales de conservación, que recomiendan la protección de al menos un $15 \%$ del área original de cada región (Burkart, 2006). Por lo tanto, es indispensable incrementar las áreas protegidas de esta región, sobre todo, por tratarse de uno de los 3 biomas de más alta diversidad de la Argentina.

\section{Agradecimientos}

Este estudio fue financiado por la Secretaría General de Ciencia y Técnica de la Universidad Nacional del Nordeste (Proyecto 17/F174) y la Agencia Nacional de Promoción Científica y Técnica, PICT o UNNE 11-0244. A Mario Favila y 2 revisores anónimos por sus comentarios y sugerencias que ayudaron a mejorar significativamente el manuscrito.

\section{Referencias}

Adámoli, J., Ginzburg, R. y Torrella, S. (2011). Escenarios productivos y ambientales del Chaco Argentino: 1977-2010. Grupo de Estudios de Sistemas Ecológicos en Ambientes Agrícolas. Fundación Producir Conservando. Universidad de Buenos Aires.

Anderson, M. J. (2005). PERMANOVA: A FORTRAN computer program for permutational multivariate analysis of variance. Auckland: Department of Statistics, University of Auckland.

Andresen, E. (2008). Dung beetle assemblages in primary forest and disturbed habitats in a tropical dry forest landscape in Western Mexico. Journal of Insect Conservation, 12, 639-650.

Arellano, L. (2002). Evaluación de la diversidad alfa, beta y gamma de Scarabaeoidea y Silphidae (Insecta: Coleoptera) en la Región Cofre de Perote, Veracruz, México. Tesis de Maestría. Facultad de Ciencias, Universidad Nacional Autónoma de México.

Barbosa-Silva, F. A., Hernández, M. I. M., Ide, S. y Moura, R. C. (2007). Comunidade de escarabeíneos (Coleoptera, Scarabaeidae) copro-necrófagos da região de Brejo Novo, Caruaru, Pernambuco Brasil. Revista Brasileira de Entomología, 51, 228-233. 
Barraza, J., Montes, J., Martínez, N. y Deloya, C. (2010). Ensamblaje de escarabajos coprófagos (Scarabaeidae: Scarabaeinae) del bosque tropical seco, Bahía Concha, Santa Marta, Colombia. Revista Colombiana de Entomología, 36, 285-291.

Bruniard, E.D. (1999). Los regímenes hídricos de las formaciones vegetales. Aportes para un modelo fotoclimático mundial. Resistencia, Chaco: Editorial Universitaria del Nordeste.

Burkart, R. (2006). Las áreas protegidas de la Argentina. En A. Brown, U. Ortiz Martínez, M. Acerba y J. Corcuera (Eds.), La situación ambiental Argentina 2005 (pp. 399-404). Buenos Aires: Fundación Vida Silvestre Argentina.

Bustos-Gómez, L. F. y Lopera-Toro, A. (2003). Preferencia por cebo de los escarabajos coprófagos (Coleoptera: Scarabaeidae: Scarabaeinae) de un remanente de bosque seco tropical al norte del Tolima (Colombia). Monografías Tercer Milenio, 3, 59-65.

Cabido, M., Molina, S. I., Pérez-Harguindeguy, N., Valladares, G., Pucheta, E. y Gardner, S. (1999). Uso de la tierra, estructura del hábitat y biodiversidad en ecosistemas del centro de la Argentina. En S. D. Matteucci, O. T. Solbrig, J. Morello y G. Halffter (Eds.), Biodiversidad y uso de la tierra. Conceptos y ejemplos de Latinoamérica (p (pp. 421-442). Buenos Aires: Editorial Universitaria de Buenos Aires.

Cabrera-Walsh, G. y Gandolfo, D. (1996). Nidification of thirteen common argentine dung beetles (Scarabaeidae: Scarabaeinae). Annals of the Entomological Society of America, 89, 581-588.

Campos, R. C. y Hernández, M. I. M. (2013). Dung beetle assemblages (Coleoptera Scarabaeinae) in Atlantic forest fragments in southern Brazil. Revista Brasileira de Entomologia, 57, 47-54.

Chao, A. y Shen, T. J. (2003). Nonparametric estimation of Shannon's index of diversity when there are unseen species in sample. Environmental and Ecological Statistics, 10, 429-433.

Chao, A. y Shen, T.J. (2010). Program SPADE (Species prediction and diversity estimation). Recuperado el 20 de diciembre de 2014 de: http://chao.stat.nthu.edu.tw/softwareCE.html

Chebez, J. C. (2005). Guía de las reservas naturales de la Argentina: nordeste. Buenos Aires: Editorial Albatros.

Clarke, K. R. y Ainsworth, M. (1993). A method linking multivariate community structure to environmental variables. Marine Ecology Progress Series, 92, 205-219.

Clarke, K. R. y Gorley, R. N. (2001). PRIMER. Version 5: User manual tutorial. Plymouth: Plymouth Marine Laboratory.

Colwell, R.K. (2006). Statistical estimation of species richness and shared species from samples (Software and user's guide).Version 8.0.Recuperado el 5 de junio de 2013 de: http://purl.oclc.org/estimates

Colwell, R. K. y Coddington, J. A. (1994). Estimating terrestrial biodiversity through extrapolation. Philosophical Transactions of the Royal Society, 345, 101-118.

Damborsky, M. P., Bar, M. E., Álvarez-Bohle, M. C. y Oscherov, E. B. (2008). Comunidad de escarabajos copronecrófagos (Coleoptera: Scarabaeidae) en dos bosques del Chaco Oriental Húmedo, Argentina. Revista de la Sociedad Entomológica Argentina, 67, 145-153.

Damborsky, M. P., Álvarez-Bohle, M. C., Ibarra-Polesel, M. G., Porcel, E. A. y Fontana, J. L. (2015). Spatial and temporal variation of dung beetle assemblages in a fragmented landscape at Eastern Humid Chaco. Neotropical Entomology, 44(6), 30-39.

Edmonds, W. D. y Zidek, J. (2010). A taxonomic review of the neotropical genus Coprophanaeus Olsoufieff 1924 (Coleoptera: Scarabaeidae, Scarabaeinae). Insecta Mundi, 129, 1-111.

Escobar, F. (2000). Diversidad y distribución de los escarabajos del estiércol (Coleoptera: Scarabaeidae: Scarabaeinae) de Colombia. En F. Martín-Piera, J. J. Morrone y A. Melic (Eds.), Hacia un proyecto CYTED para el inventario y estimación de la diversidad entomológica en Iberoamérica: PrIBES-2000 (pp. 197-210). Zaragoza: Monografías Tercer Milenio.

Escobar, M. J., Ávalos, G. y Damborsky, M. P. (2012). Diversidad de Araneae (Arachnida) en la Reserva Colonia Benítez Chaco Oriental Húmedo. Argentina. Facena, 28, 3-17.

Favila, M. E. (2005). Diversidad alfa y beta de los escarabajos del estiércol (Scarabaeinae) en Los Tuxtlas México. En G. Halffter, J. Soberón, P. Koleff y A. Melic (Eds.), Sobre diversidad biológica: el significado de las diversidades alfa, beta y gamma (pp. 209-219). Zaragoza: M3mmonografías tercer milenio.

Favila, M. E. y Halffter, G. (1997). The use of indicator groups for measuring biodiversity as related to community structure and function. Acta Zoológica Mexicana n.s., 72, 1-25.

Génier, F. (1996). A revision of the Neotropical genus Ontherus Erichson. Memoirs of the Entomological Society of Canada, 170, 1-169.

Génier, F. (2009). Le genre Eurysternus Dalman, 1824 révision taxonomique et clés de détermination illustrées. Pensoft Series Faunistica, 85, 1-430.

Gill, B. D. (1991). Dung beetles in tropical American forests. En I. Hanski e Y. Camberfort (Eds.), Dung beetle ecology (pp. 211-229). Princeton: Princeton University Press.

Ginzburg, G. R. y Adámoli, J. (2006). Situación ambiental en el Chaco Húmedo. En A. Brown, U. Ortiz Martínez, M. Acerbi y J. Corcuera (Eds.), La situación ambiental Argentina 2005 (pp. 103-113). Buenos Aires: Fundación Vida Silvestre Argentina.

Godoy, M. C., Laffont, E. R., Coronel, J. M. y Etcheverry, C. (2013). Termite (Insecta Isoptera) assemblage of a gallery forest relic from the Chaco province (Argentina): taxonomic and functional groups. Arxius de Miscellània Zoològica, 10, 55-67.

Gómez-Lutz, M. C. y Godoy, M. C. (2010). Diversidad y grupos funcionales de Formicidae (Insecta, Hymenoptera) de la reserva natural educativa Colonia Benítez (provincia del Chaco, Argentina). Revista FABICIB, 14, 180-195.

Gotelli, N. J. y Chao, A. (2013). Measuring and estimating species richness, species diversity and biotic similarity from sampling data. En S. A. Levin (Ed.), Encyclopedia of biodiversity. (5) (2nd ed., 5, pp. 195-211). Waltham: Academic Press.

Halffter, G. (1991). Historical and ecological factors determining the geographical distribution of beetles (Coleoptera: Scarabaeidae: Scarabaeinae). Folia Entomológica Mexicana, 82, 195-238.

Halffter, G. y Edmonds, W. D. (1982). The nesting behavior of dung beetles (Scarabaeinae) an ecologic and evolutive approach. México, D.F: Man and Biosphere Program UNESCO.

Halffter, G. y Favila, M. E. (1993). The Scarabaeinae (Insecta: Coleoptera) an animal group for analyzing, inventorying and monitoring biodiversity in tropical rainforest and modified landscapes. Biology International, 27 , $15-21$.

Halffter, G., Favila, M. E. y Halffter, V. (1992). A comparative study of the structure of the scarab guild in Mexican tropical rain forests and derived ecosystems. Folia Entomológica Mexicana, 84, 131-156.

Halffter, G. y Halffter, V. (2009). Why and where coprophagous beetles (Coleoptera: Scarabaeinae) eat seeds, fruits or vegetable detritus. Boletín de la Sociedad Entomológica Aragonesa, 45, 1-22.

Halffter, G. y Matthews, E. G. (1966). The natural history of dung beetles of the subfamily Scarabaeinae (Coleoptera, Scarabaeidae). Folia Entomológica Mexicana, 12, 1-312.

Hanski, I. y Cambefort, Y. (1991). Dung beetle ecology. Princeton: Princeton University Press.

Heinonen-Fortabat, S. y Chebez, J. C. (1997). Los mamíferos de los parques nacionales de la Argentina. Buenos Aires: Editorial L.O.L.A.

Hernández, M. I. M. y Vaz de Mello, F. Z. (2009). Seasonal and spatial species richness variation of dung beetle (Coleoptera Scarabaeidae s. str.) in the Atlantic Forest of southeastern Brazil. Revista Brasileira de Entomologia, 53, 607-613

Janzen, H. D. (1983). Seasonal changes in abundance of large nocturnal dung beetles (Scarabaeidae) in a Costa Rica deciduous forest and adjacent horse pastures. Oikos, 33, 274-283.

Jiménez-Valverde, A. y Hortal, J. (2003). Curvas de acumulaciónde especies y la necesidad de evaluar la calidad de los inventarios biológicos. Revista Ibérica de Aracnología, 8, 151-161.

Kremen, C., Colwell, R. K., Erwin, T. L., Murphy, D. D., Noss, R. F. y Saujayan, M. A. (1993). Terrestrial arthropod assemblages: Their use as indicators in conservation planning. Conservation Biology, 7, 796-808.

Jost, L. (2006). Entropy and diversity. Oikos, 113, 363-375.

Ledesma, L. L. (1995). Los suelos de la estación experimental agropecuaria Colonia Benítez «Dr. Agusto G. Schulz» Provincia del Chaco. Chaco, Argentina: Instituto Nacional de Tecnología Agropecuaria, E.E. A. Pres. Roque Sáenz Peña. 
Martínez, N. J. (2010). Coleópteros coprófagos (Scarabaeidae: Scarabaeinae) en un fragmento de bosque seco tropical en el departamento del Atlántico Colombia. Boletín del Museo de Entomología de la Universidad del Valle, $11,21-30$.

Martínez, N. J., García, H., Pulido, L. A., Ospino, D. D. y Narváez, J. C. (2009). Escarabajos coprófagos (Coleoptera: Scarabaeinae) de la vertiente noroccidental, sierra Nevada de Santa Marta, Colombia. Neotropical Entomology, 38, 708-715.

Martínez-Hernández, N., Muñoz, G. S., Sierra-Quintero, K. y Barraza-Méndez, J. (2012). Escarabajos coprófagos (Coleoptera: Scarabaeinae) asociados a excrementos de mamíferos en un fragmento de bosque seco tropical en el departamento del Atlántico, Colombia. Ecología Austral, 22, 203-210.

Mc Geoch, M. A., Sithole, H., Samways, M. J., Simaika, J. P., Pryke, J. S., Picker, M., et al. (2011). Conservation and monitoring of invertebrates in terrestrial protected áreas. Koedoe, 53, 1-13.

Milhomem, M., Vaz de Melo, F. Z. y Diniz, I. R. (2003). Técnicas de coleta de besouros copronecrófagos no cerrado. Pesquisa Agropecuária Brasileira, 38, 1249-1256.

Moreno, C. E., Barragán, F., Pineda, E. y Pavón, N. P. (2011). Reanálisis de la diversidad alfa: alternativas para interpretar y comparar información sobre comunidades ecológicas. Revista Mexicana de Biodiversidad, 82, 1249-1261.

Nichols, E., Larsen, T., Spector, S., Davis, A., Escobar, F., Favila, M. E., et al. (2007). Global dung beetle response to tropical forest modification and fragmentation: A quantitaive review and meta-analysis. Biological Conservation, 137, 1-19.

Nichols, E., Spector, S., Louzada, J., Larsen, T., Amezquita, S. y Favila, M. E. (2008). Ecological functions and ecosystem services provided by Scarabaeinae dung beetles. Biological Conservation, 141, 1461-1474.

Oliver, I. y Beattie, A. J. (1993). A possible method for the rapid assessment of biodiversity. Conservation Biology, 7, 562-568.

Orozco, J. y Pérez, M. (2008). Escarabajos coprófagos (Coleoptera, Scarabaeoidea) del Parque Nacional Los Estoraques (Norte de Santander Colombia). Revista Brasileira de Entomologia, 52, 36-40.
Sackmann, P. (2006). Efectos de la variación temporal y los métodos de captura en la eficiencia de un muestreo de coleópteros en la Reserva Natural Loma del Medio, El Bolsón, Río Negro. Revista de la Sociedad Entomológica Argentina, 65, 35-50.

Schnack, J. A. y López, H. L. (2003). Biodiversidad, iniciativa global y elaboración de Inventarios sistemáticos. ProBiota, serie Técnica y Didáctica, 3, 1-14.

Schulz, A.G. (1957). Reserva Biológica de Colonia Benítez. Catálogo de la flora de la selva. Estación experimental agropecuaria Colonia Benítez. INTA. Centro Regional Chaqueño. Boletín n. ${ }^{\circ} 2$.

Shen, T. J., Chao, A. y Lin, J. F. (2003). Predicting the number of new species in further taxonomic sampling. Ecology, 84, 798-804.

Silva, P. G. y di Mare, R. A. (2012). Escarabeíneos copro-necrófagos (Coleoptera, Scarabaeidae, Scarabaeinae) de fragmentos de Mata Atlântica em Silveira Martins, Rio Grande do Sul Brasil. Iheringia, série Zoologia, 102, 197-205

Silva, P. G., Rosa-García, M. A. y Vidal, M. B. (2009). Adultos de Scarabaeidae sensu stricto (Coleoptera) coletados em áreas naturais de campo e de floresta, em Bagé, RS. Revista de Ciências Agroveterinárias, 8, 62-65.

Silva, P. G., Rosa-García, A. M. y Vidal, M. B. (2009). Besouroscopronecrófagos (Coleoptera: Scarabaeidae sensu stricto) do Município de Bagé, RS (Bioma Campos Sulinos). Biociências, 17, 33-43.

Silva, P. G., Vaz de Mello, F. Z. y di Mare, R. A. (2012). Attractiveness of different bait to the Scarabaeinae (Coleoptera: Scarabaeidae) in forest fragments in extreme Southern Brazil. Zoological Studies, 5, 429-441.

Spector, S. (2006). Scarabaeinae dung beetles (Coleoptera: Scarabaeidae: Scarabaeinae): an invertebrate focal taxon for biodiversity research and conservation. Coleopterists Bulletin, 60, 71-83.

Vaz de Mello, F. Z., Edmonds, W. D., Ocampo, F. C. y Schoolmeesters, P. (2011). A multilingual key to the genera and subgenera of the subfamily Scarabaeinae of the New World (Coleoptera: Scarabaeidae). Zootaxa, 2854, $1-73$. 